\title{
Epidemiological Study of Collar Rot Disease of Indian Bean caused by Sclerotium rolfsii in South Gujarat
}

\author{
Tushar V. Ghevariya* and P.R. Patel \\ Department of Plant Pathology, NM College of Agriculture, Navsari Agricultural University, \\ Navsari - 396 450, Gujarat, India \\ *Corresponding author
}

\section{A B S T R A C T}

\section{Keywords}

Epidemiology, Collar rot, Indian bean, Correlation, Regression, Weather Parameter

Article Info

Accepted:

04 October 2019

Available Online:

10 November 2019
Collar rot of Indian bean caused by Sclerotium rolfsii Sacc. is exerting a major threat on Indian bean growers in South Gujarat. The initiation of the disease was first noticed after 10 and 15 days of sowing in susceptible variety GNIB-21 which was grown on $11^{\text {st }}$ Oct. and $12^{\text {nd }}$ Oct. of 2017-18 and 2018-19, respectively. Since then, there was linear progress of the disease during both the seasons. The disease appeared $2.6 \%$ at initial stage $44^{\text {th }}$ SMW and reached highest $37.2 \%$ at maturity $3{ }^{\text {rd }}$ SMW during 201718. In 2018-19, disease appeared $2.75 \%$ at initial stage $44^{\text {th }}$ SMW and reached highest $38.47 \%$ at maturity $3^{\text {rd }}$ SMW. However in pooled of two years, the first symptom of collar rot was recorded on $44^{\text {th }}$ SMW $(2.6 \%)$ and reached to $3^{\text {rd }}$ SMW $(37.2 \%)$. Correlation coefficient study between weather parameters and disease incidence indicated that all the parameters jointly played an important role in the development of collar rot disease. Moreover, morning and afternoon $5 \mathrm{~cm}$ and $10 \mathrm{~cm}$ depth soil temperature, soil moisture $5 \mathrm{~cm}$ and $10 \mathrm{~cm}$ were found to play important role in the disease development. Co-efficient of determination, $\mathrm{R}^{2}$-value i.e., $0.675,0.954$ and 0.881 percent were found high during 2018, 2019 and pooled of two year, respectively. These high $\mathrm{R}^{2}$-value indicating a strong association between percent disease incidence to weather parameters.

\section{Introduction}

Indian bean (Lablab purpureus L.) belonging to family Fabaceae, is one of the most ancient cultivated crops and is presently grown throughout the tropical regions in Asia, Africa and America. It is commonly known as Hyacinth bean, Dolichos bean, Avare (Kannada), Anumulu (Telugu), Avaria (Tamil), Indian bean, lablab bean, sembean, lobia bean (Sudan), wal-papdi (Gujarat) and Egyptian Kidney bean. Indian bean is affected by several biotic and abiotic stresses. Among several fungal biotic stresses, diseases like Ashy stem blight (Macrophomina phaseolina), rust (Uromyces fabae), Powdery mildew (Erysiphe polygoni), Anthracnose (Colletotrichum lindemuthianum), Bacterial leaf spot (Xanthomonas campestris), Bean common mosaic virus, Sclerotinia blight 
(Sclerotinia sclerotiorum) Melouk and Backman, 1995. Rhizoctonia limb, pod and root rot, Sclerotium stem rot (Collar rot) are the major constraints for Indian bean cultivation. Among these, collar rot caused by $S$. rolfsii is an important disease. The fungus is polyphagous, ubiquitous, omnivorous and soil borne in nature, having wide host range which resist adverse environmental condition, hence it is very difficult to control. $S$. rolfsii widely distributed in tropics, subtropics and also in warmer parts of temperate zone of the world. In India, it is wide spread in almost all the states causing economic losses in many crops. The numerous reports from tropical and sub tropical areas of the world, coupled with the large number of hosts attacked by it indicate that, economic losses are substantial every year due to infection of $S$. rolfsii (Aycock, 1966). Among the several biotic factors contributing towards low production of chickpea, the collar rot disease caused by $S$. rolfsii is a major cause when weather conditions are conducive. The disease is favored by good soil moisture, high soil temperature $\left(25-30^{\circ} \mathrm{C}\right)$ and low organic matter in the soil (Mathur and Sinha, 1968). Singh and Dwivedi (1987) proposed disease incidence with soil moisture and soil temperature while, a maximum of 72 per cent disease incidence was recorded at high relative humidity and moderate temperature in case of collar rot of pigeon pea incited by $S$. rolfsii. Therefore, the present studies were done to know the effect of different weather parameter on the collar rot disease incidence of Indian bean.

\section{Materials and Methods}

The experiment was conducted at N. M. College of Agriculture farm, NAU, Navsari, during rabi 2017-18 and rabi 2018-19. It is $20.95^{\circ}$ North latitude (N) and $72.93^{\circ}$ East longitude (E) and altitude is 12.33 meter above sea level. Epidemiological study on the effect of weather parameters on the collar rot disease of Indian bean in the fixed plot was $14 \mathrm{~m} \times 12 \mathrm{~m}$ and at a distance of $60 \mathrm{~cm} \times 30 \mathrm{~cm}$ cultivar GNIB-21 was used. Fertilizers and other agronomical practices were followed according to the recommendations for growing high-yielding varieties. To determine the influence of various environmental factors on the development of collar rot of Indian bean, the disease incidence data were correlated with different meteorological parameters viz., maximum temperature (Max. Temp.), minimum temperature (Min. Temp.), average temperature (Av. Temp.) morning relative humidity (RH1), afternoon relative humidity (RH2), average relative humidity, rainfall (RF), sunshine ( $\mathrm{SH})$, soil temperature, rainy days, soil $\mathrm{pH}$ and soil moisture through analysis of correlation and regression. The weekly observation on collar rot incidence was recorded throughout the season. Correlation and regression equation between disease incidence dependent factors were worked out using the standard procedure given by Gomez and Gomez (1984).

The weekly observation on collar rot incidence were recorded for two cropping seasons of the year right from the initiation of disease to maturity stage of the crop. Data of weather parameters corresponding to disease observation were collected from meteorological department, N. M. College of Agriculture, NAU, Navsari. GNIB-21 variety was selected for the studies and 44 plants were randomly selected and were observed on the collar rot disease development. Observations were recorded at the initiation of the disease and thereafter at weekly interval of standard meteorological week. Per cent disease incidence was calculated using formula proposed by Wheeler (1969).

Per cent disease incidence (PDI)

PDI $=$ No. of diseased plants X 100/Total No of plants observed 


\section{Soil temperature}

Soil temperature was recorded by the soil thermometer installed in the plot at weekly interval. The soil thermometer was inserted in soil at 5 and $10 \mathrm{~cm}$ depth as suggested by Nwankwol and Ogagarue (2012).

\section{Soil moisture (soil oven dry weight method)}

Soil samples were collected at weekly interval. Such five samples from different sites (four corner and one center of the plot) were taken at a depth of 5 and $10 \mathrm{~cm}$ and composite sample was prepared from the plot for epidemiological study. Fresh weight of soil and weight of oven dry soil were recorded. The soil moisture content was determined as follows (Black, 1965).

$\mathrm{PW}(\%)=\frac{\mathrm{WS} 1-\mathrm{WS} 2}{\mathrm{WS} 2} \times 100$

Where,

$\mathrm{PW}=$ Moisture per cent on dry weight basis

WS1 $=$ Weight of fresh soil $(\mathrm{g})$

WS2 $=$ Weight of oven dried soil $(\mathrm{g})$

\section{Results and Discussion}

\section{Progress of collar rot in cv. GNIB-21 under natural condition}

The observations on collar rot incidence were recorded at a weekly interval in cv. GNIB-21 at N. M. college farm, Navsari during the year of 2017-18 and 2018-19 which was presented in Table 1, 2 and 3.

The first symptom of collar rot was recorded 10 to15 days after sowing in susceptible variety GNIB-21. Which was sown on 11 October of 2017 and harvesting on 23 January
2017. Since then, there was linear progress of the disease during the entire crop season. The main peak period of the collar rot was recorded at 44 SMW to 52 SMW (2.42 to $30.38 \%$ ) and reached at its highest on 3 SMW (35.97 \%) during 2017-18. Whereas in 201819 was sown on 12 October of 2018 and harvesting on 25 January 2018. Since then, there was linear progress of the disease during the entire crop season. The main peak period of the collar rot was recorded, at 44 SMW to 52 SMW (2.75 to $31.44 \%)$ and reached at its highest on 3 SMW (38.47\%) in 2018-19. However, in pooled of two years symptom of collar rot was 44 SMW to 3 SMW linear progress of the disease during the entire crop season.

The disease at the starting period $(2.60 \%)$ to reached $(37.20 \%)$. Comparatively, collar rot incidence was more during 2018-19 than in 2017-18. The weather during 48 to 51 SMW played an important role in initiation of the collar rot. During 1 to $3 \mathrm{SMW}$, there was very fast development of the disease due to congenial condition.

Hence, this period can be considered as a window period for the collar rot of Indian bean in Navsari condition. Collar rot infection starts from 44 to 45 SMW under favourable weather conditions. The infection observed dark brown lesion on the stem just below the soil surface followed by drooping and wilting of infected leaves and gradually wilting of the whole plant in the presence of favourable climatic conditions, resulted yield loss.

From the results of the present investigation, it is very clear that collar rot of Indian bean is very important and become a major disease in Navsari district. Hence, it causes heavy losses because, its infection starts at all growth stage including the germinating stage of the seed causing pre-emergence rot and young plant shown stem rot so, it directly effects on yield. 
Correlation of collar rot incidence with weather parameters

The disease started appearing at young stage of the crop and epidemiological studies were performed right up to harvesting of the crop. The collar rot infection in susceptible cv. GNIB-21 and corresponding weather parameters at weekly interval were recorded which was presented in Table 4. During 2017, collar rot was initiated on 44 SMW. Previous two weeks 42 and 43 SMW were recorded maximum temperature i.e., 35.9 and $35.0^{\circ} \mathrm{C}$, average temperature i.e., 29.1 and $27.4^{\circ} \mathrm{C}$, morning relative humidity i.e., 92.8 and 90.3 percent, bright sunshine hours i.e., 7.2 and 9.3 hrs. and soil temperature morning $5 \mathrm{~cm}$ i.e., 22.0 and 21.0, respectively. These conditions favoured for initiation of the collar rot during seedling stage. More dominant favourable weather conditions $v i z$, maximum temperature i.e., $29.5, \quad 29.8$ and $31.1^{\circ} \mathrm{C}$, average temperature i.e., $22.5,23.2$ and $21.1^{\circ} \mathrm{C}$, morning relative humidity i.e., $92.3,63.1$ and 82.2 percent, bright sunshine hours i.e., 6.4, 3.8 and $8.1 \mathrm{hrs}$. and soil temperature morning $5 \mathrm{~cm} \mathrm{26.5,} \mathrm{27.5,} 27.0$ lead to more progress of the disease during 50, 51 and 52 SMW, respectively. There was a continuously increased of the collar rot during 50 to 52 SMW in the presence of the warm and humid weather which considered a window period for the collar rot of Indian bean. After 52 SMW up to harvest, the disease incidence was continuously increased and recorded up to 35.97 percent disease incidence. Correlation matrix worked out showed that maximum temperature (-0.754), minimum temperature ($0.642)$ and average temperature $(-0.822)$, were found highly significantly and negatively correlated with the collar rot incidence, respectively. Moreover, morning soil temperature $5 \mathrm{~cm} \quad(0.701)$, morning soil temperature $10 \mathrm{~cm}(0.746)$, afternoon soil temperature $10 \mathrm{~cm}(0.629)$, soil moisture $5 \mathrm{~cm}$ (0.797), and soil moisture $10 \mathrm{~cm}(0.819)$ was found highly significant and positive correlated with the collar rot incidence. Morning soil temperature $5 \mathrm{~cm}$, morning soil temperature $10 \mathrm{~cm}$, afternoon soil temperature $10 \mathrm{~cm}$, soil moisture $5 \mathrm{~cm}$, and soil moisture $10 \mathrm{~cm}$ were playing an impotent role in the disease development compared to other weather parameters. More or less similar trends were recorded during the subsequent year in the progress of the collar rot due to closely similar weather. In 2018, the disease was initiated on 44 SMW. Previous two weeks 42 and 43 SMW were recorded maximum temperature i.e., 36.4 and $36.8^{\circ} \mathrm{C}$, average temperature i.e., 28.4 and $26.8^{\circ} \mathrm{C}$, morning relative humidity i.e., 81.6 and 78.6 percent, bright sunshine hours i.e., 8.5 and $10.0 \mathrm{hrs}$. and soil temperature morning $5 \mathrm{~cm}$ i.e., 20.7 and 21.3, respectively. These conditions favoured for initiation of the collar rot during seedling stage. More dominant favourable weather conditions viz., maximum temperature i.e., $\quad 30.0, \quad 29.7$ and $25.2^{\circ} \mathrm{C}, \quad$ average temperature i.e., $21.7,20.0$ and $16.9^{\circ} \mathrm{C}$, morning relative humidity i.e., $86.7,80.8$ and 71.2 percent, bright sunshine hours i.e., 7.2, 8.8 and $7.1 \mathrm{hrs}$. and soil temperature morning $5 \mathrm{~cm} \mathrm{21.7,} \mathrm{20.0,} 16.9$ lead to more progress of the disease during 50, 51 and 52 SMW, respectively. After 52 SMW up to harvest, the disease incidence was continuously increased and recorded up to 38.47 percent disease incidence. Correlation matrix worked out showed that maximum temperature $(-0.880)$, minimum temperature (-0.617), average temperature (-0.797) afternoon relative humidity $(-0.774)$, average relative humidity (0.652 ) and bright sun shine hours (-0.657) were found highly significantly and negatively correlated with the collar rot incidence, respectively. Moreover, afternoon soil temperature $5 \mathrm{~cm}$ (0.837), afternoon soil temperature $10 \mathrm{~cm}(0.630)$, soil moisture $5 \mathrm{~cm}$ (0.693), and soil moisture $10 \mathrm{~cm}(0.720)$ was found highly significant and positive correlated with the collar rot incidence. 
Morning soil temperature $5 \mathrm{~cm}$, morning soil temperature $10 \mathrm{~cm}$, afternoon soil temperature $10 \mathrm{~cm}$, soil moisture $5 \mathrm{~cm}$, and soil moisture $10 \mathrm{~cm}$ were playing an impotent role in the disease development compared to other weather parameters. The results clearly reveal that there is two years that the collar rot become a rising problem in Navsari district because of the presence of a pathogen, growing susceptible cv.GNIB-21 in most of the area and prevalence of congenial weather conditions. During mean of two years, collar rot was initiated on 44 SMW. Previous two weeks 42 and 43 SMW were recorded maximum temperature i.e., 36.1 and $35.9^{\circ} \mathrm{C}$, average temperature i.e., 28.7 and $27.1^{\circ} \mathrm{C}$, morning relative humidity i.e., 87.2 and 84.5 percent, bright sunshine hours i.e., 7.9 and 9.7 hrs. and soil temperature morning $5 \mathrm{~cm}$ i.e., 21.4 and 21.2, respectively. These conditions favoured for initiation of the collar rot disease during seedling stage. More dominant favourable weather conditions viz., maximum temperature i.e., 29.8, 29.7 and $28.2^{\circ} \mathrm{C}$, average temperature i.e., 22.1, 21.6 and $19.0^{\circ} \mathrm{C}$, morning relative humidity i.e., 89.5 , 71.9 and 76.7 percent, bright sunshine hours i.e., $6.8,6.3$ and $7.6 \mathrm{hrs}$. and soil temperature morning $5 \mathrm{~cm} \mathrm{26.8,27.3,} 27.2$ lead to more progress of the disease during 50, 51 and 52 SMW, respectively. After $52 \mathrm{SMW}$ up to harvest, the disease incidence was continuously increased and recorded up to 37.20 percent disease incidence. Correlation matrix worked out showed that maximum temperature (-0.885), minimum temperature (0.666), average temperature (-0.832) and bright sun shine hours (-0.553) were found highly significantly and negatively correlated with the collar rot incidence, respectively.
Moreover, Morning soil temperature $5 \mathrm{~cm}$ (0.606), Morning soil temperature $10 \mathrm{~cm}$ (0.616), afternoon soil temperature $5 \mathrm{~cm}$ (0.564), afternoon soil temperature $10 \mathrm{~cm}$ (0.647), soil moisture $5 \mathrm{~cm}(0.747)$ and soil moisture $10 \mathrm{~cm}(0.777)$ was found highly significant and positive correlated with the collar rot incidence. Morning soil temperature $5 \mathrm{~cm}$, morning soil temperature $10 \mathrm{~cm}$, afternoon soil temperature $10 \mathrm{~cm}$, soil moisture $5 \mathrm{~cm}$, and soil moisture $10 \mathrm{~cm}$ were playing an impotent role in the disease development compared to other weather parameters. Step wise regression equation for Indian bean collar rot on different weather parameters at Navsari. The regression coefficient based on stepwise regression analysis for percent collar rot incidence of Indian bean with respect to weather parameters have been worked out and presented in Table 5, 6 and 7. The result presented in Table 5 indicated, stepwise regression, it has been also observed that Rvalue was high $(0.675 \%)$ indicating a strong association between percent disease incidence to average temperature. This clearly indicates that at least 67 percent of variation in collar rot incidence can be explained by the function of the weather parameter average temperature during 2017. The result presented in Table 6 indicated, stepwise regression, it has been also observed that R-value was high (0.954\%) indicating a strong association between percent disease incidence to maximum temperature average, afternoon relative humidity and soil $\mathrm{pH}$. This clearly indicates that at least 95.4 percent of the variation in collar rot incidence can be explained by the function of the weather parameter viz., maximum temperature average, afternoon relative humidity and soil pH during 2018. 
Table.1 Meteorological parameters with collar rot incidence on cv. GNIB-21 during Rabi -2017-18

\begin{tabular}{|c|c|c|c|c|c|c|c|c|c|c|c|c|c|c|c|c|c|}
\hline SMW & PDI & $\begin{array}{c}\mathbf{T} \\
\mathbf{M a x} \\
\left({ }^{\circ} \mathbf{C}\right)\end{array}$ & $\begin{array}{c}\mathbf{T} \\
\mathbf{M i n} \\
\left({ }^{\circ} \mathbf{C}\right)\end{array}$ & $\begin{array}{l}\text { Av. } \\
\text { Tem } \\
\left({ }^{\circ} \mathrm{C}\right)\end{array}$ & Soi & tem & Soi & tem & mo & $\begin{array}{l}\text { il } \\
\text { ture } \\
\text { o) }\end{array}$ & & $\%)$ & $\begin{array}{l}\text { Av. } \\
\text { RH } \\
(\%)\end{array}$ & $\begin{array}{c}\text { BSSH } \\
\text { (hrs) }\end{array}$ & $\begin{array}{c}\text { RF } \\
(\mathbf{m m})\end{array}$ & $\begin{array}{c}\text { Soil } \\
\text { pH }\end{array}$ & $\begin{array}{c}\text { Rainy } \\
\text { day }\end{array}$ \\
\hline & & & & & $5 \mathrm{~cm}$ & $10 \mathrm{~cm}$ & $5 \mathrm{~cm}$ & $10 \mathrm{~cm}$ & $5 \mathrm{~cm}$ & $10 \mathrm{~cm}$ & & & & & & & \\
\hline & & & & & I & I & II & II & & & I & II & & & & & \\
\hline 42 & 0 & 35.9 & 22.3 & 29.1 & 22.0 & 22.5 & 23.0 & 24.0 & 27.1 & 24.3 & 92.8 & 72.0 & 82.4 & 7.2 & 0.0 & 7.0 & 0.0 \\
\hline 43 & 0 & 35.0 & 19.8 & 27.4 & 21.0 & 22.0 & 23.5 & 24.0 & 27.0 & 25.0 & 90.3 & 46.8 & 68.6 & 9.3 & 0.0 & 7.1 & 0.0 \\
\hline 45 & 4.11 & 34.3 & 16.3 & 25.3 & 23.0 & 23.0 & 24.5 & 26.0 & 27.9 & 25.7 & 72.1 & 31.2 & 51.7 & 9.6 & 0.0 & 7.1 & 0.0 \\
\hline 46 & 6.22 & 32.3 & 14.3 & 23.3 & 22.5 & 24.0 & 23.5 & 24.5 & 29.1 & 27.1 & 84.3 & 31.4 & 57.8 & 8.3 & 0.0 & 7.2 & 0.0 \\
\hline 47 & 9.39 & 34.0 & 15.7 & 24.9 & 24.0 & 24.5 & 25.0 & 25.0 & 30.3 & 27.4 & 87.8 & 39.8 & 63.8 & 6.5 & 0.0 & 7.1 & 0.0 \\
\hline 48 & 13.61 & 33.1 & 15.0 & 24.1 & 26.5 & 27.0 & 27.5 & 28.0 & 32.1 & 29.9 & 74.3 & 33.7 & 54.0 & 8.0 & 0.0 & 7.2 & 0.0 \\
\hline 49 & 17.82 & 26.6 & 17.9 & 22.3 & 25.0 & 27.0 & 28.0 & 28.0 & 33.6 & 30.4 & 83.7 & 68.3 & 76.0 & 2.5 & 5.3 & 7.3 & 2.0 \\
\hline 50 & 21.63 & 29.5 & 15.6 & 22.5 & 26.5 & 28.0 & 29.5 & 30.0 & 34.6 & 32.7 & 92.3 & 48.0 & 70.1 & 6.4 & 0.0 & 7.3 & 0.0 \\
\hline 51 & 26.48 & 29.8 & 16.6 & 23.2 & 27.5 & 28.0 & 29.0 & 30.0 & 36.7 & 33.8 & 63.1 & 40.8 & 51.9 & 3.8 & 0.0 & 7.2 & 0.0 \\
\hline 52 & 30.38 & 31.1 & 11.0 & 21.1 & 27.0 & 29.0 & 29.0 & 29.5 & 38.8 & 35.2 & 82.2 & 33.4 & 57.8 & 8.1 & 0.0 & 7.2 & 0.0 \\
\hline 1 & 32.69 & 29.7 & 10.2 & 20.0 & 27.0 & 27.5 & 27.0 & 30.5 & 36.4 & 35.3 & 90.2 & 34.7 & 62.5 & 7.9 & 0.0 & 7.1 & 0.0 \\
\hline 2 & 34.6 & 29.5 & 15.6 & 22.6 & 24.0 & 25.0 & 24.5 & 25.0 & 34.1 & 30.4 & 64.2 & 35.9 & 50.1 & 5.8 & 0.0 & 7.0 & 0.0 \\
\hline 3 & 35.97 & 31.1 & 15.0 & 23.1 & 24.0 & 25.5 & 25.0 & 26.0 & 30.1 & 28.8 & 80.9 & 33.2 & 57.1 & 7.6 & 0.0 & 7.1 & 0.0 \\
\hline
\end{tabular}

$\mathrm{PDI}=$ per cent disease incidence $\quad \mathrm{RH}=$ relative humidity $\quad$ BSSH= bright sun shine hours $\quad \mathrm{I}=$ Morning $\mathrm{II}=$ Afternoon 
Table.2 Meteorological parameters with collar rot incidence on cv. GNIB-21 during Rabi-2018-19

\begin{tabular}{|c|c|c|c|c|c|c|c|c|c|c|c|c|c|c|c|c|c|}
\hline \multirow[t]{2}{*}{ SMW } & \multirow[t]{2}{*}{ PDI } & \multirow[t]{2}{*}{$\begin{array}{c}\mathbf{T} \\
\mathrm{Max} \\
\left({ }^{\circ} \mathrm{C}\right)\end{array}$} & \multirow[t]{2}{*}{$\begin{array}{c}\text { T } \\
\text { Min } \\
\left({ }^{\circ} \mathbf{C}\right)\end{array}$} & \multirow[t]{2}{*}{$\begin{array}{l}\text { Av. } \\
\text { Tem } \\
\left({ }^{\circ} \mathrm{C}\right)\end{array}$} & \multicolumn{2}{|c|}{$\begin{array}{l}\text { Soil tem } \\
\left({ }^{\circ} \mathrm{C}\right)\end{array}$} & \multicolumn{2}{|c|}{$\begin{array}{l}\text { Soil tem } \\
\quad\left({ }^{\circ} \mathrm{C}\right)\end{array}$} & \multicolumn{2}{|c|}{$\begin{array}{c}\text { Soil } \\
\text { moisture } \\
(\%)\end{array}$} & \multicolumn{2}{|c|}{ RH(\%) } & \multirow[t]{2}{*}{$\begin{array}{l}\text { Av. } \\
\text { RH } \\
(\%)\end{array}$} & \multirow[t]{2}{*}{ BSSH } & \multirow[t]{2}{*}{$\begin{array}{c}\text { RF } \\
(\mathbf{m m})\end{array}$} & \multirow[t]{2}{*}{$\begin{array}{c}\text { Soil } \\
\text { pH }\end{array}$} & \multirow[t]{2}{*}{$\begin{array}{c}\text { Rainy } \\
\text { day }\end{array}$} \\
\hline & & & & & $5 \mathrm{~cm}$ & $10 \mathrm{~cm}$ & $5 \mathrm{~cm}$ & $10 \mathrm{~cm}$ & $5 \mathrm{~cm}$ & $10 \mathrm{~cm}$ & & & & & & & \\
\hline & & & & & I & I & II & II & & & I & II & & & & & \\
\hline 42 & 0 & 36.4 & 20.3 & 28.4 & 20.7 & 21.1 & 22.0 & 23.0 & 26.1 & 25.1 & 81.6 & 58.0 & 69.8 & 8.5 & 0.0 & 7.1 & 0.0 \\
\hline 43 & 0 & 36.8 & 16.7 & 26.8 & 21.3 & 21.9 & 22.4 & 23.6 & 26.7 & 25.3 & 78.6 & 49.3 & 64.0 & 10.0 & 0.0 & 7.1 & 0.0 \\
\hline 44 & 2.75 & 35.5 & 16.9 & 26.2 & 22.5 & 23.2 & 23.0 & 23.5 & 26.2 & 25.0 & 75.1 & 50.0 & 62.6 & 10.0 & 0.0 & 7.2 & 0.0 \\
\hline 45 & 4.15 & 35.5 & 14.8 & 25.2 & 24.4 & 25.0 & 25.0 & 25.0 & 27.9 & 26.2 & 90.8 & 58.5 & 74.7 & 9.0 & 0.0 & 7.1 & 0.0 \\
\hline 46 & 6.38 & 35.0 & 13.5 & 24.3 & 24.0 & 25.2 & 24.8 & 25.1 & 28.6 & 27.0 & 86.6 & 57.7 & 72.2 & 8.7 & 2.0 & 7.2 & 0.0 \\
\hline 47 & 9.6 & 35.3 & 16.3 & 25.8 & 23.2 & 25.0 & 26.0 & 27.2 & 31.2 & 28.0 & 91.3 & 55.4 & 73.4 & 8.0 & 0.0 & 7.1 & 0.0 \\
\hline 48 & 13.75 & 33.6 & 13.9 & 23.8 & 25.0 & 26.1 & 26.4 & 27.5 & 32.6 & 30.1 & 80.1 & 50.5 & 65.3 & 7.6 & 0.0 & 7.3 & 0.0 \\
\hline 49 & 18.24 & 31.9 & 13.2 & 22.6 & 26.3 & 26.9 & 26.5 & 30.0 & 34.0 & 31.8 & 88.0 & 53.5 & 70.8 & 5.9 & 0.0 & 7.1 & 0.0 \\
\hline 50 & 22.08 & 30.0 & 13.4 & 21.7 & 27.0 & 27.4 & 27.0 & 30.6 & 36.4 & 32.7 & 86.7 & 52.9 & 69.8 & 7.2 & 0.0 & 7.2 & 0.0 \\
\hline 51 & 27.53 & 29.7 & 10.3 & 20.0 & 27.1 & 28.6 & 29.0 & 31.5 & 38.6 & 35.1 & 80.8 & 54.1 & 67.5 & 8.8 & 0.0 & 7.3 & 0.0 \\
\hline 52 & 31.44 & 25.2 & 8.6 & 16.9 & 27.3 & 28.0 & 29.2 & 32.6 & 38.7 & 35.0 & 71.2 & 46.5 & 58.9 & 7.1 & 0.0 & 7.3 & 0.0 \\
\hline 1 & 34.01 & 29.7 & 10.2 & 20.0 & 24.0 & 24.6 & 27.0 & 28.8 & 36.6 & 34.1 & 90.2 & 34.7 & 62.5 & 7.9 & 0.0 & 7.2 & 0.0 \\
\hline 2 & 36.89 & 29.5 & 15.6 & 22.6 & 23.4 & 24.0 & 24.2 & 26.0 & 32.2 & 30.1 & 64.2 & 35.9 & 50.1 & 5.8 & 0.0 & 7.2 & 0.0 \\
\hline 3 & 38.47 & 31.1 & 15.0 & 23.1 & 22.7 & 23.4 & 24.0 & 26.2 & 29.1 & 27.5 & 80.9 & 33.2 & 57.1 & 7.6 & 0.0 & 7.1 & 0.0 \\
\hline
\end{tabular}

$\mathrm{PDI}=$ per cent disease incidence $\quad \mathrm{RH}=$ relative humidity $\quad$ BSSH= bright sun shine hours $\quad \mathrm{I}=$ Morning $\mathrm{II}=$ Afternoon 
Table.3 Meteorological parameters with collar rot incidence on cv. GNIB-21 during Rabi Pooled

\begin{tabular}{|c|c|c|c|c|c|c|c|c|c|c|c|c|c|c|c|c|c|}
\hline \multirow[t]{2}{*}{ SMW } & \multirow[t]{2}{*}{ PDI } & \multirow[t]{2}{*}{$\begin{array}{c}\text { T } \\
\text { Max } \\
\left({ }^{\circ} \mathbf{C}\right)\end{array}$} & \multirow[t]{2}{*}{$\begin{array}{c}\text { T } \\
\text { Min } \\
\left({ }^{\circ} \mathbf{C}\right)\end{array}$} & \multirow[t]{2}{*}{$\begin{array}{l}\text { Av. } \\
\text { Tem } \\
\left({ }^{\circ} \mathrm{C}\right)\end{array}$} & \multicolumn{2}{|c|}{$\begin{array}{l}\text { Soil tem } \\
\left({ }^{\circ} \mathrm{C}\right)\end{array}$} & \multicolumn{2}{|c|}{$\begin{array}{c}\text { Soil tem } \\
\left({ }^{\circ} \mathrm{C}\right)\end{array}$} & \multicolumn{2}{|c|}{$\begin{array}{c}\text { Soil } \\
\text { moisture } \\
(\%)\end{array}$} & \multicolumn{2}{|c|}{ RH(\%) } & \multirow[t]{2}{*}{$\begin{array}{l}\text { Av. } \\
\text { RH } \\
(\%)\end{array}$} & \multirow[t]{2}{*}{$\begin{array}{c}\text { BSSH } \\
\text { (hrs) }\end{array}$} & \multirow[t]{2}{*}{$\begin{array}{c}\mathbf{R F} \\
(\mathbf{m m})\end{array}$} & \multirow[t]{2}{*}{$\begin{array}{l}\text { Soil } \\
\text { pH }\end{array}$} & \multirow[t]{2}{*}{$\begin{array}{c}\text { Rainy } \\
\text { day }\end{array}$} \\
\hline & & & & & $5 \mathrm{~cm}$ & $10 \mathrm{~cm}$ & $5 \mathrm{~cm}$ & $10 \mathrm{~cm}$ & $5 \mathrm{~cm}$ & $10 \mathrm{~cm}$ & & & & & & & \\
\hline & & & & & I & I & II & II & & & I & II & & & & & \\
\hline 42 & 0.0 & 36.1 & 21.3 & 28.7 & 21.4 & 21.8 & 22.5 & 23.5 & 26.6 & 24.7 & 87.2 & 65.0 & 76.1 & 7.9 & 0.0 & 7.2 & 0.0 \\
\hline 43 & 0.0 & 35.9 & 18.3 & 27.1 & 21.2 & 22.0 & 23.0 & 23.8 & 26.9 & 25.2 & 84.5 & 48.0 & 66.3 & 9.7 & 0.0 & 7.1 & 0.0 \\
\hline 44 & 2.6 & 35.4 & 16.9 & 26.2 & 22.5 & 23.1 & 23.5 & 23.8 & 26.9 & 25.1 & 73.6 & 36.7 & 55.1 & 9.9 & 0.0 & 7.2 & 0.0 \\
\hline 45 & 4.1 & 34.9 & 15.5 & 25.2 & 23.7 & 24.0 & 24.8 & 25.5 & 27.9 & 26.0 & 81.5 & 44.9 & 63.2 & 9.3 & 0.0 & 7.1 & 0.0 \\
\hline 46 & 6.3 & 33.7 & 13.9 & 23.8 & 23.3 & 24.6 & 24.2 & 24.8 & 28.9 & 27.1 & 85.4 & 44.5 & 65.0 & 8.5 & 1.0 & 7.2 & 0.0 \\
\hline 47 & 9.5 & 34.7 & 16.0 & 25.3 & 23.6 & 24.8 & 25.5 & 26.1 & 30.8 & 27.7 & 89.6 & 47.6 & 68.6 & 7.3 & 0.0 & 7.1 & 0.0 \\
\hline 48 & 13.7 & 33.4 & 14.5 & 23.9 & 25.8 & 26.6 & 27.0 & 27.8 & 32.4 & 30.0 & 77.2 & 42.1 & 59.6 & 7.8 & 0.0 & 7.2 & 0.0 \\
\hline 49 & 18.0 & 29.2 & 15.6 & 22.4 & 25.7 & 27.0 & 27.3 & 29.0 & 33.8 & 31.1 & 85.8 & 60.9 & 73.4 & 4.2 & 2.6 & 7.1 & 1.0 \\
\hline 50 & 21.9 & 29.8 & 14.5 & 22.1 & 26.8 & 27.7 & 28.3 & 30.3 & 35.5 & 32.7 & 89.5 & 50.4 & 70.0 & 6.8 & 0.0 & 7.3 & 0.0 \\
\hline 51 & 27.0 & 29.7 & 13.5 & 21.6 & 27.3 & 28.3 & 29.0 & 30.8 & 37.7 & 34.5 & 71.9 & 47.5 & 59.7 & 6.3 & 0.0 & 7.2 & 0.0 \\
\hline 52 & 30.9 & 28.2 & 9.8 & 19.0 & 27.2 & 28.5 & 29.1 & 31.1 & 38.8 & 35.1 & 76.7 & 39.9 & 58.3 & 7.6 & 0.0 & 7.3 & 0.0 \\
\hline 1 & 33.4 & 29.7 & 10.2 & 20.0 & 25.5 & 26.1 & 27.0 & 29.7 & 36.5 & 34.7 & 90.2 & 34.7 & 62.5 & 7.9 & 0.0 & 7.2 & 0.0 \\
\hline 2 & 35.7 & 29.5 & 15.6 & 22.6 & 23.7 & 24.5 & 24.4 & 25.5 & 33.2 & 30.3 & 64.2 & 35.9 & 50.1 & 5.8 & 0.0 & 7.1 & 0.0 \\
\hline 3 & 37.2 & 31.1 & 15.0 & 23.1 & 23.4 & 24.5 & 24.5 & 26.1 & 29.6 & 28.2 & 80.9 & 33.2 & 57.1 & 7.6 & 0.0 & 7.1 & 0.0 \\
\hline
\end{tabular}

PDI= per cent disease incidence $\quad \mathrm{RH}=$ relative humidity $\quad \mathrm{BSSH}=$ bright sun shine hours $\quad \mathrm{I}=$ Morning II= Afternoon 
Table.4 Relationship of collar rot incidence with different weather variables during 2017-18 and 2018-19 (pooled)

\begin{tabular}{|c|c|c|c|c|}
\hline \multirow{2}{*}{$\begin{array}{l}\text { Sr. } \\
\text { No. }\end{array}$} & \multirow[t]{2}{*}{ Weather variables } & \multicolumn{3}{|c|}{ Correlation Coefficient " $r$ " } \\
\hline & & Rabi- 2017 & Rabi -2018 & Pooled \\
\hline 1 & Maximum temperature $\left({ }^{0} \mathrm{C}\right)$ & $-0.754 * *$ & $-0.880 * *$ & $-0.885 * *$ \\
\hline 2 & Minimum temperature $\left({ }^{0} \mathrm{C}\right)$ & $-0.642 * *$ & $-0.617 * *$ & $-0.666 * *$ \\
\hline 3 & Average temperature $\left({ }^{0} \mathrm{C}\right)$ & $-0.822 * *$ & $-0.797 * *$ & $-0.832 * *$ \\
\hline 4 & Morning soil temperature $5 \mathrm{~cm}\left({ }^{0} \mathrm{C}\right)$ & $0.701 * *$ & 0.455 & $0.606 * *$ \\
\hline 5 & Morning soil temperature $10 \mathrm{~cm}\left({ }^{0} \mathrm{C}\right)$ & $0.746^{* *}$ & 0.423 & $0.616 * *$ \\
\hline 6 & Afternoon soil temperature $5 \mathrm{~cm}\left({ }^{0} \mathrm{C}\right)$ & $0.525^{*}$ & $0.537 * *$ & $0.564 * *$ \\
\hline 7 & Afternoon soil temperature $10 \mathrm{~cm}\left({ }^{0} \mathrm{C}\right)$ & $0.629 * *$ & $0.630 * *$ & $0.647 * *$ \\
\hline 8 & Soil moisture $5 \mathrm{~cm}$ & $0.797 * *$ & $0.693 * *$ & $0.747 * *$ \\
\hline 9 & Soil moisture $10 \mathrm{~cm}$ & $0.819 * *$ & $0.720 * *$ & $0.777 * *$ \\
\hline 10 & Morning relative humidity (\%) & -0.245 & -0.300 & -0.313 \\
\hline 11 & Afternoon relative humidity (\%) & -0.202 & $-0.774 * *$ & $-0.510 *$ \\
\hline 12 & Average relative humidity (\%) & -0.255 & $-0.652 * *$ & $-0.494 *$ \\
\hline 13 & Bright sun shine hours & -0.403 & $-0.657 * *$ & $-0.553 * *$ \\
\hline 14 & Rainfall (mm) & 0.22 & -0.226 & -0.067 \\
\hline 15 & Soil pH & -0.008 & 0.073 & 0.130 \\
\hline 16 & Rainy days & 0.022 & 0.00 & 0.017 \\
\hline
\end{tabular}

Note: Critical value $(0.05)=+/-0.458^{*}$ and $0.532 * *$

Table.5 Regression $\left(\mathrm{R}^{2}\right)$ analysis of disease incidence of collar rot with different meteorological parameter in 2017-18

\begin{tabular}{|c|c|c|c|c|}
\hline $\begin{array}{c}\text { Sr. } \\
\text { no. }\end{array}$ & Independent variables & Intercept & $\begin{array}{c}\text { Regression }\left(\mathbf{R}^{\mathbf{2}}\right) \\
\text { coefficient 'b' }\end{array}$ & $\mathbf{R}^{\mathbf{2}}$ \\
\hline 1. & $\mathrm{X}_{3}=$ average temperature & 124.501 & -4.499 & 0.675 \\
\hline
\end{tabular}

Table.6 Regression $\left(\mathrm{R}^{2}\right)$ analysis of disease incidence of collar rot with different meteorological parameter in 2018-19

\begin{tabular}{|c|c|c|c|c|}
\hline $\begin{array}{l}\text { Sr. } \\
\text { no }\end{array}$ & Independent variables & Intercept & $\begin{array}{c}\text { Regression }\left(\mathbf{R}^{2}\right) \\
\text { coefficient ' } b \text { ' }\end{array}$ & $\mathbf{R}^{2}$ \\
\hline 1. & $\mathrm{X}_{1}=$ Maximum temperature & \multirow{3}{*}{445.740} & -3.004 & \multirow{3}{*}{0.954} \\
\hline 2. & $\mathrm{X}_{11}=$ Afternoon relative humidity & & -0.693 & \\
\hline 3. & $\mathrm{X}_{15}=$ Soil $\mathrm{pH}$ & & -41.369 & \\
\hline
\end{tabular}

Table.7 Regression $\left(\mathrm{R}^{2}\right)$ analysis of disease incidence of collar rot with different meteorological parameter (Pooled analysis)

\begin{tabular}{|c|c|c|c|c|}
\hline $\begin{array}{c}\text { Sr. } \\
\text { no. }\end{array}$ & Independent variables & Intercept & $\begin{array}{c}\text { Regression }\left(\mathbf{R}^{\mathbf{2}}\right) \\
\text { coefficient 'b' }\end{array}$ & $\mathbf{R}^{\mathbf{2}}$ \\
\hline $\mathbf{1 .}$ & $\mathrm{X}_{1}=$ Maximum temperature & 163.663 & -3.879 & 0.881 \\
\hline $\mathbf{2}$ & $\mathrm{X}_{11}=$ Afternoon relative humidity & & -0.476 & \\
\hline
\end{tabular}


The result presented in Table 7 indicated, stepwise regression, it has been also observed that R-value was high $(0.881 \%)$ indicating a strong association between percent disease incidence to maximum temperature and Afternoon relative humidity. This clearly indicates that at least 88.1 percent of variation in collar rot incidence can be explained by the function of the weather parameter viz., maximum temperature and afternoon relative humidity during pooled of two years. Thus, observed collar rot incidence and predicted collar rot incidence found closely related and regression equation established may be most reliable and useful for forecasting of the collar rot of Indian bean. The loss caused by the collar rot can be saved by forewarning to the farmers and thereby controlling the same at the proper time. The result was support with many workers. According to finding more or less similar with Dalvi and Raut (1986) found that the optimum temperature and relative humidity required for $S$. rolfsii of groundnut. Sahu and Narain (1995) reported viability of sclerotia at up to $80 \%$ soil moisture level with $60-70 \%$ being optimum. Sclerotial viability was adversely affected at saturated soil moisture levels. Pinheiro et al., (2010) observed intermediate soil moisture level (70\% of field capacity) and temperatures ranging between $25-30^{\circ} \mathrm{C}$ favoured sclerotial development. Increasing soil temperature and soil moisture was increased mycelium growth of $S$. rolfsii and viability of sclerotia in collar rot of Indian bean.

\section{References}

Aycock, R. A. (1966). Stem rot and other diseases caused by Sclerotium rolfsii. Tech. Bull. 174. Agric. Expt. Station, North Carolina State University, Raleigh. 202.

Black, C. A. (1965). Methods of soil analysis: part I physical and mineralogical properties. American Society of
Agronomy, Madison, Wisconsin, USA. pp76. (Fide: https://dl.sciencesocieties. org/publications/books/articles/agrono mymonogra/methodsofsoilana/ frontmatter).

Dalvi, M. B. and Raut, S. P. (1986). Effect of temperature and humidity on growth and sclerotia formation of Sclerotium rolfsii causing groundnut wilt. Indian Journal of Mycology and Plant Pathology, 16(2): 175-176.

Gomez K. and Gomez A.A. (1984) John Wiley and Sons, New York. p. 680.

Mathur, S. B. and Sinha, S. (1968). Disease development in guar (Cyamopsis psoraloides DC.) and gram (Cicer arietinum L.) attacked with Sclerotium rolfsii under different soil conditions. Phytopathology, 62: 319-322.

Melouk, H. A. and Backman, P. A. (1995). Management of soil borne fungal pathogens. Pages 75-82.

Nwankwol, C. and Ogagarue, D. (2012). An investigation of temperature variation at soil depths in parts of Southern Nigeria. American Journal of Environmental Engineering, 2(5): 142147.

Pinheiro, V. R., Seixas, C. D. S., Godoy, C. V., Soares, R.M., Oliveira, M. C. N. and Almeida, A. M. R. (2010). Development of Sclerotium rolfsii sclerotia on soybean, corn, and wheat straw, under different soil temperatures and moisture contents. Pesquisa Agropecuaria Brasileira, 45 (3): 332334.

Sahu, K. C. and Narain, A. (1995). Environment and ecology of Sclerotium rolfsii in soil at different temperature and moisture levels. Environment and Ecology, 13(2): 300303.

Singh, R. K. and Dwivedi, R. S. (1987). Effect of different chemicals on morphological changes in Sclerotium 
rolfsii causing foot rot of barley. Plant Disease, John Wiley and Sons Indian Phytopathology, 40: 112-113. Limited, London, pp: 301.

Wheeler, B. E. J. (1969). An Introduction of

\section{How to cite this article:}

Tushar V. Ghevariya and Patel, P.R. 2019. Epidemiological Study of Collar Rot Disease of Indian Bean caused by sclerotium rolfsii in South Gujarat. Int.J.Curr.Microbiol.App.Sci. 8(11): 193-203. doi: https://doi.org/10.20546/ijcmas.2019.811.024 